\title{
REVIEW
}

\section{Year in review 2012: Critical Care - nephrology}

\author{
Zaccaria Ricci ${ }^{{ }^{*}}$ and Claudio Ronco ${ }^{2,3}$
}

\begin{abstract}
We summarize original research in the field of critical care nephrology accepted or published in 2012 in Critical Care and, when considered relevant or directly linked to this research, in other journals. Three main topics have been identified for a rapid overview: acute kidney injury, detailed in some pathogenetic and epidemiological aspects; fluid overload as a predictor of mortality both in acute kidney injury and renal replacement therapy (RRT) patients; and RRT, evaluating some features of citrate anticoagulation and describing the effects of RRT modalities or timing on survival.
\end{abstract}

\section{Acute kidney injury 2012 Intrarenal hemodynamics}

Acute kidney injury (AKI) pathogenesis is currently widely debated and intense scientific efforts are being made to better elucidate mechanisms of renal damage. In particular, renal perfusion is one of the most investigated aspects of septic AKI pathogenesis: both hypoperfusion and renal vasodilatation with increased renal blood flow (RBF) have been considered by recent experimental and human studies on AKI $[1,2]$.

The easiest way to assess renal perfusion at the bedside is to measure renal vascular resistance by Doppler. Interestingly, Dewitte and coworkers tested the hypothesis that mean arterial pressure is a determinant of the renal resistive index (RI) in septic and critically ill patients who do or do not have AKI [3]. Intrarenal Doppler signals were obtained from two or three proximal interlobar arteries in 94 critically ill septic patients with stable hemodynamic conditions: of these, about 50\% were AKI patients (in one-half of these cases, transient AKI was diagnosed) and 50\% were receiving norepinephrine. The authors found that: median renal RIs were slightly higher in patients with AKI than in those

\footnotetext{
* Correspondence: z.ricci@libero.it

'Department of Pediatric Cardiosurgery, Bambino Gesù Children's Hospital,

IRCCS, Piazza S. Onofrio 4, 00165, Rome, Italy

Full list of author information is available at the end of the article
}

without; RIs were similar between transient and persistent AKI; RIs did not differ in patients receiving norepinephrine infusion and were not correlated with norepinephrine dose; and only in patients without AKI were RIs inversely correlated with mean arterial pressure and partial pressure of arterial oxygen:fraction of inspired oxygen ratio, whereas they had direct correlation with age. The authors honestly conclude that, in the light of their results, renal circulatory response to sepsis cannot be reliably predicted by a single Doppler ultrasonography. On the other hand, interestingly this work confirms that, in septic critically ill patients without AKI, renal perfusion increases with pressure and is probably associated with reduced renal vascular resistance, whereas renal vasoconstriction seen in AKI patients seems unresponsive to hemodynamic support; however, due to the poor value of such correlations, the authors insist that renal RIs have a multifactorial regulation and no definitive conclusion can be drawn by this study on the exact mechanism determining renal perfusion autoregulation in septic patients.

Interestingly a comprehensive review on this field by Schnell and Darmon reached the same conclusions and recommended a large, adequately powered study in nonselected patients before implementing this technique in clinical practice [4]. One must clarify, however, that flow velocity is measured by Doppler ultrasound, not perfusion; the concept that increased or decreased RI corresponds to increased or decreased vascular resistance is speculative. As a general rule, one must highlight that RBF can only be estimated by Doppler.

Bragadottir and coauthors were also interested in studying intrarenal hemodynamics, and they conducted an interventional study on 11 post-cardiosurgical patients with signs of AKI [5]. They introduced a catheter, in the postoperative phase, into the left renal vein in order to measure RBF with retrograde thermodilution: $15 \%$ mannitol infusion was used to test renal hemodynamics. They found in this cohort of patients that mannitol induced a significant increase in RBF (12\%) and significantly decreased renal vascular resistance $(-13 \%)$. Mannitol also caused significant increases in urine output (61\%) and in fractional excretion of 
sodium (58\%). Mannitol tended to increase the glomerular filtration rate $(16 \%)$ and renal vein oxygenation $(10 \%)$ without reaching statistical significance. Interestingly, due to the scarce effect of mannitol on cardiac output, the authors found that it increased the RBF/cardiac output relation. They conclude that, other than the wellknown effect of osmotic diuresis, mannitol induced a renal vasodilatation and increased RBF with no changes in filtration fraction or the renal oxygen supply/demand relation, thus somehow redistributing systemic blood flow to the kidneys; these data maybe of some value in all cases where a RBF decrease is suspected.

\section{Burn-associated and contrast-induced acute kidney injury} Continuing along the path of AKI pathogenesis, it must be remarked, once again, that critically ill patients undergo different types of AKI and that it is very important to recognize exact AKI etiology in order to optimize its management [6]. A particularly interesting and rather overlooked form of renal dysfunction is that occurring in severely burned patients [7]. An important difference for burn-associated AKI is the intensity and duration of the inflammatory response, which may be persistent, especially when a significant portion of total burned surface area (namely $>30 \%$ ) is present [7]. Septic renal dysfunction, multiorgan failure and thrombogenic factors are considered risk factors for direct renal parenchymal damage in severely burned patients [8]. Typically, proteinuria occurs in burned patients, mirroring a clinical and subclinical glomerular and tubular damage [7]. $\mathrm{Hu}$ and coworkers interestingly analyzed a retrospective cohort of 396 severely burned patients; of these, $68.43 \%$ had proteinuria and more than one-half of them met AKI criteria [7]. Remarkably, no patient without proteinuria developed AKI. In the light of this study, the amount of protein loss in urine not only seemed a clear index of clinical and subclinical renal injury, but was also proportional to ICU mortality. This study suggests that proteinuria should be used for identifying burn patients at risk of developing AKI and those with worse prognosis.

Another form of AKI addressed last year by Critical Care authors was contrast-induced nephropathy (CIN), which typically refers to a decline in the glomerular filtration rate occurring in the first days following contrast media administration for any radiological procedure. Interestingly, Cely and coworkers examined CIN risk factors in critically ill patients matching those who underwent computed tomography (CT) scan with and without contrast media [9]. Pairs of patients were matched on prescan measured creatinine clearance, vasopressor use, mechanical ventilation, and diabetes mellitus. Different from other studies, predominantly focused on patients undergoing cardiac catheterization, the authors found that the principal outcome - a 33\% decrease in measured creatinine clearance - declined similarly in contrast patients (26\%) and in noncontrast patients (36\%) and that the average peak decline in clearance was $19 \pm 29 \%$ among contrast patients and 26 $\pm 39 \%$ among noncontrast patients. The authors conclude reporting that severity of critical illness (leading to the need for CT) rather than contrast media is responsible for the described declines in renal function. These results are further supported by the fact that contrast patients with lowest prescan creatinine clearance still did not show any significant difference in post-CT AKI incidence with respect to their noncontrast pairs. Finally, within the evaluated cohort, one patient per group underwent RRT and only two noncontrast patients died.

What it is also interesting about this study is that several prophylactic measures for CIN were undertaken in some of the examined patients; $\mathrm{N}$-acetylcysteine, bicarbonate and additional pre-emptive fluids were given in $79 \%$ of contrast patients, but none of these methods revealed significant improvement of post-CT-scan renal function. Of note, after decades of debate on prevention of CIN, meta-analyses still do not provide significantly effective measures, in most cases advocating significantly larger trials than those currently performed [10]. As suggested by Cely and colleagues, critically ill patients undergoing administration of contrast media should be enrolled in most of these studies to definitely clarify the additional CIN risk in this high risk of AKI population [11].

\section{Classification}

The interesting conclusion of this subgroup of studies published in Critical Care about AKI is that all of them defined AKI according to a standardized definition. The Acute Dialysis Quality Initiative, back in 2004, published the consensus definition of the Risk, Injury, Failure, Loss of function, End-stage kidney disease (RIFLE) criteria [12]; this acronym conventionally described three AKI stages of progressively higher creatinine levels or decreased urine output flows (Risk, Injury, Failure) and two outcomes (Loss of function and End-stage kidney disease). The Acute Kidney Injury Network subsequently introduced small but important modifications to this classification [13]. The RIFLE and the Acute Kidney Injury Network criteria are now, finally, a universal tool to be used in AKI research studies.

Probably for this reason, only one study addressed the issue of AKI definition/classification last year. Wlodzimirow and coauthors described in detail the importance of applying urine output (RIFLE-UO) criteria together with creatinine criteria (RIFLE-Cr) when AKI epidemiology is evaluated [14]. In a prospective cohort study on 260 patients, these authors applied both RIFLE-Cr and 
RIFLE-UO + Cr and realized that only the second criterion allows a correct estimation of AKI incidence, maximum RIFLE grade distribution, and AKI timely diagnosis (in 24 patients, AKI diagnosis was anticipated by the application of RIFLE-UO $+\mathrm{Cr}$ ). Interestingly they found that the mortality rate in the AKI population was significantly lower when based on RIFLE-Cr + UO; probably the higher sensitivity of the complete RIFLE classification also includes less severely ill patients with transient renal dysfunction, but gives a more realistic view of AKI severity and prognosis [15]. Interestingly, in Wlodzimirow and colleagues' study, all AKI patients showed a rather positive cumulative fluid balance; even if no significant differences between the diagnostic criteria were found, the RIFLE-Cr + UO criteria seemed to display less positive values - it is possible that urine output decrease immediately triggers fluid infusion restriction, hence reducing the amount of excess fluids.

Recently, the issue of AKI classification has been reappraised by the Kidney Disease: Improving Global Outcomes (KDIGO) workgroup, who published the first international, interdisciplinary clinical practice guideline on AKI [16], which is also available on the KDIGO website [17]. The aim of KDIGO was also to finally resume all currently used AKI classifications and to find a unique definitive standardized staging [18].

\section{Fluid balance: are we paying enough attention?}

Two papers discussed the influence of fluid management on mortality of critically ill patients in 2012; one focused on the effects of fluid overload on changes in creatinine levels [19], and the other addressed the subject of fluid balance optimization in RRT patients [20]. Both papers calculated the percentage of fluid accumulation by dividing the cumulative balance by the patient's baseline weight and multiplying by 100 . Stein and collaborators [19] evaluated prospectively a cohort of 502 post-cardiac surgery patients over a 12-month period; of these, $3.4 \%$ did not survive. They realized that fluid overload $>10 \%$ increased the risk of death by 1.6 per each standardized step ( $Z$ score) of fluid accumulation; similarly, a creatinine increase $>0.3 \mathrm{mg} / \mathrm{dl}$ with respect to baseline also showed a significant effect, the risk of death being more than doubled per each $Z$-score change. Interestingly, the authors also tried to adjust serum creatinine levels over a fluid balance correction factor, due to the potential of creatinine hemodilution in overloaded patients [21], but did not find significant changes in creatinine risk prediction. Fluid overload also clearly impacted the length of ICU stay, showing that ventilation time and days needed for a complete recovery, in cardiac surgery patients, takes advantage from a lower fluid intake. In this light, by analyzing complicated cases (those staying in the ICU $>4$ days) they found that surviving patients were all fluid negative whereas $75 \%$ of nonsurvivors had fluid excess $>10 \%$. Unfortunately, this study did not directly clarify the relationship between AKI diagnosis and creatinine increase and between fluid overload and urine output decrease. Furthermore, only seven patients of this cohort underwent RRT (we have no information on their fluid balance) and it is not clear from these results whether an aggressive management of fluid balance might have resulted in improved outcomes.

Vaara and the FINNAKI study group seemed to seek an answer regarding the effects of RRT management of fluid overload; from a prospective data collection of 283 patients undergoing RRT, patients with fluid overload at dialysis start had twice as high crude 90-day mortality compared with those without fluid overload $(59 \%$ vs. $31 \%$ ) [20]. These results were confirmed even after adjustments for disease severity, time of RRT initiation, initial RRT modality and sepsis. Interestingly, $75 \%$ of patients received continuous RRT (CRRT), fluidoverloaded patients were mostly the septic ones, in only $45 \%$ of times was the indication to RRT specifically fluid accumulation, and a delay in RRT start was clearly associated with a progressive positivization of fluid balance. From this observational study the importance of fluid management before RRT initiation and its significant impact on subsequent outcomes clearly emerge. Unfortunately again, however, the authors do not report data on fluid balance during RRT days and whether forcing fluid download might have a role in patients' prognosis. This important subject was previously addressed by Selewski and co-workers in pediatric patients undergoing extracorporeal membrane oxygenation and CRRT [22]. According to these authors, there is no way to improve a patient's outcome once fluid overload is accumulated in extracorporeal membrane oxygenation children before CRRT start. The authors conclude by suggesting an early CRRT start in extracorporeal membrane oxygenation patients if the fluid balance is not adequately controlled. However, a recent post-hoc analysis of the huge Randomised Evaluation of Normal vs. Augmented Level of Replacement Therapy trial, conducted on CRRT adults (without extracorporeal membrane oxygenation), showed instead that a mean negative cumulative fluid balance achieved in the first 48 hours after CRRT start did improve the outcome of critically ill patients [23]. One must acknowledge that fluid-overloaded patients are also probably the sickest, and one should note that, currently, fluid administration per kilogram of body weight has to be limited in the first phases of critically ill patient resuscitation and that aggressive diuretic therapy coupled with proactive extracorporeal ultrafiltration start might contributing to improving patients' outcomes $[24,25]$. 


\section{Renal replacement therapy: unsolved issues Regional citrate anticoagulation}

Anticoagulation with citrate and RRT modality are certainly the most important questions currently raised by expert investigators in the field of critical care nephrology.

As far as citrate is concerned, three groups examined specific aspects of this regional anticoagulation technique in 2012. Regional citrate anticoagulation (RCA) is an effective and safe alternative to heparin; citrate is infused into the extracorporeal circuit and chelates ionized calcium $\left(\mathrm{Ca}^{\text {ion }}\right)$, thereby inhibiting coagulation. Morabito and coauthors interestingly proposed a simplified citrate protocol adopting a commercially available, lowconcentration citrate solution $(0.33 \%)$ in critically ill patients with a high bleeding risk undergoing CRRT for AKI following cardiac surgery [26]. The choice of using a low-concentration citrate solution derives from the concept of simplifying the setup, by using citrate as a predilution reinfusion solution (average flow rate of $1.69 \mathrm{l} /$ hour) actively participating in the overall dialytic dose. The authors further simplified the RCA scheme, avoiding the use of dialytic or postfilter reinfusion solution without calcium; a calcium-containing postdilution replacement fluid $(1.75 \mathrm{mmol} / \mathrm{l})$ at an average rate of $0.8 \mathrm{l} /$ hour was administered after the filter, thus completing the dialytic dose requirements and reducing the need for calcium chloride supplementation (necessary to restore calcium levels after citrate chelation) (Figure 1). The authors took advantage of a mathematical model to roughly estimate citratemia and the calcium balance in order to further simplify the initial RRT prescription.
Another merit of the described protocol was that the estimated citrate load was below the lowest range reported until now by other authors and prevented citrate accumulation. However, on the other hand, the low administration of citrate was associated with a suboptimal buffer supply, with the need for additional bicarbonate infusion in more than $80 \%$ of cases. Even if simplified, this method still requires accurate and frequent blood-level checks of circuit calcemia, systemic calcemia (ionized and total), systemic bicarbonate and base excess levels, and potassium, phosphate and magnesium levels.

Two studies on RCA investigated the issue of the ionized calcium to total calcium ratio ( $\mathrm{T} / \mathrm{I} \mathrm{Ca}^{2+}$ ) $[27,28]$. This ratio is a quite important issue in RCA; in fact, despite removal of up to $50 \%$ of the citrate by the dialyzer as a complex bound with $\mathrm{Ca}^{\text {ion }}$, a certain amount of citrate enters the systemic circulation. Citrate is predominantly metabolized in the hepatic citric acid cycle leading to the release of $\mathrm{Ca}^{\text {ion }}$ into the systemic circulation and contributing to the supply of alkaline plasma buffer bases, because $1 \mathrm{~g}$ citrate produces $3 \mathrm{~g}$ bicarbonate. Nonmetabolized citrate instead chelates $\mathrm{Ca}^{\text {ion }}$, leading again to a decrease in its concentrations. $\mathrm{CaCl}_{2}$ is continuously infused in the systemic circulation when RCA is performed, in order to achieve a steady state between citrate administration by central infusion and citrate elimination determined by liver metabolism. Once a steady-state citrate concentration is achieved, a normal $\mathrm{Ca}^{\text {ion }}$ concentration can be achieved by an increased total calcium concentration because a fraction of the $\mathrm{Ca}^{\text {ion }}$ is chelated by circulating systemic citrate; hence, the $\mathrm{T} / \mathrm{I} \mathrm{Ca}^{2+}$ ratio is directly proportional to the

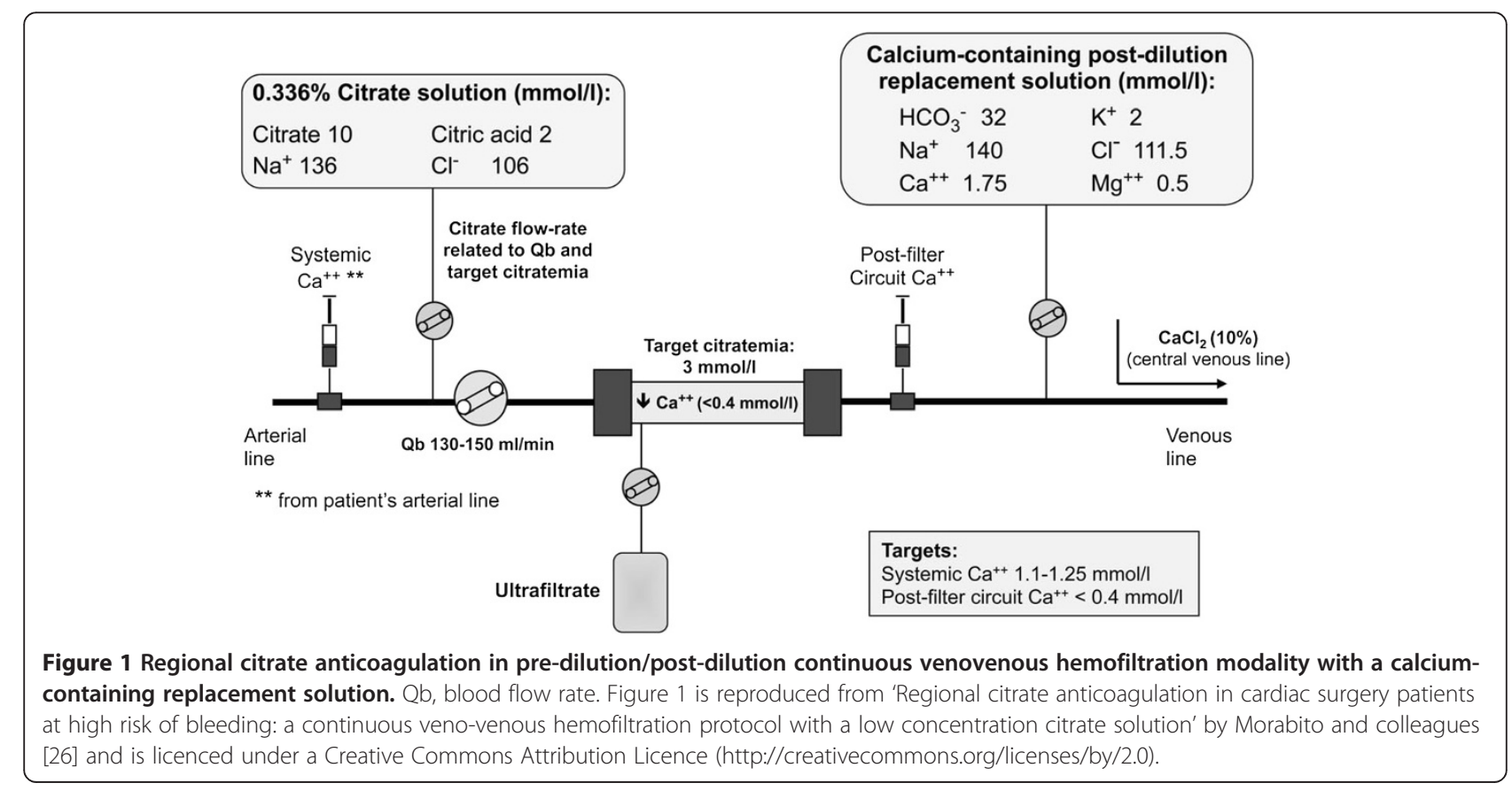


concentration of serum citrate $[27,28]$ : impaired hepatic citrate metabolism leads to citrate accumulation and increases the $\mathrm{T} / \mathrm{I} \mathrm{Ca}^{2+}$ ratio with normal $\mathrm{Ca}^{\text {ion }}$ [29]. The $\mathrm{T} / \mathrm{I} \mathrm{Ca}^{2+}$ ratio was analyzed with similar purposes by Schultheiß and coworkers [27] in patients with liver failure and by Link and colleagues [28] in patients with multiple organ dysfunction syndrome. Using a $4 \%$ citrate solution, Schultheiß realized that the threshold $\mathrm{T} / \mathrm{I} \mathrm{Ca}^{2+}$ ratio of 2.5 could be exceeded in patients with liver dysfunction without major disturbances of acid-base and electrolyte status, and correlated significantly with plasmatic citrate levels; according to these authors, RCA application in hepatic failure patients is feasible through constant monitoring of the $\mathrm{T} / \mathrm{I} \mathrm{Ca}{ }^{2+}$ ratio [27]. In particular, the authors showed that the crucial threshold of $\mathrm{T} / \mathrm{I} \mathrm{Ca}^{2+}$ ratio $\geq 2.5$ was predicted by a serum lactate level $\geq 3.4 \mathrm{mmol} / \mathrm{l}$ and a prothrombin time $\leq 26 \%$, whereas other indices of liver failure such as aspartate aminotransferase, alanine aminotransferase, bilirubin, cholinesterase, the Child-Pugh score, the Model of Endstage Liver Disease score and the plasma disappearance rate for indocyanine green did not show any significant correlation with $\mathrm{T} / \mathrm{I} \mathrm{Ca}{ }^{2+}$. The importance of the $\mathrm{T} / \mathrm{I} \mathrm{Ca}^{2+}$ ratio was confirmed by Link and coworkers, who analyzed a cohort of 203 critically ill patients undergoing CRRT with RCA (also using $4 \%$ citrate); when dividing their population into tertiles of $\mathrm{T} / \mathrm{I} \mathrm{Ca}^{2+}$ ratio $(<2.00,2.00$ to 2.39 , and 2.40 or more), the authors found that severity of liver disease, norepinephrine dose and CRRT duration, rather than age and diagnosis or severity of critical illness score, were significantly higher in the last tertile [28]. Furthermore, T/I $\mathrm{Ca}^{2+}$ ratio $>2.4$ was significantly associated with a more than 30-fold higher risk of 28-day mortality. In critically ill patients with multiorgan failure, the $\mathrm{T} / \mathrm{I} \mathrm{Ca}^{2+}$ ratio appears a specific prognostic biomarker and, similarly to that described in liver failure patients, a threshold of 2.4 seems to reveal severely impaired hepatic function and should not be exceeded when RCA is used.

\section{Modality, timing, indications}

Important works have been published on the most debated issues of RRT delivery and their impact on patient outcome: the modality of therapy administration, the timing of therapy inception and, finally, the indication of RRT. In particular, an interesting and provocative feasibility study - the Optimal Mode of clearance in critically ill patients with Acute Kidney Injury - was conducted [30]; the effects of convective (continuous venovenous hemofiltration) versus diffusive (continuous venovenous hemodialysis) solute clearance on clinical outcomes (change in Sequential Organ Failure Assessment score from baseline to days 1,2 and 7) were compared. The prescribed dialytic dose was set at $35 \mathrm{ml} / \mathrm{kg} / \mathrm{hour}$ and, when delivered via hemofiltration, adjusted in cases of predilution replacement solution infusion. From a clinical standpoint, the two rather small groups of about 30 critically ill patients showed a nonsignificant tendency for Sequential Organ Failure Assessment improvement, due to the hemodynamic component, in the continuous venovenous hemofiltration group. The primary aim of the study, however, was to verify whether administration of $>75 \%$ of the prescribed CRRT dose to participants in each treatment arm was feasible. Secondary feasibility outcomes included the ability to enroll $>25 \%$ of fully eligible patients and the ability to follow $>95 \%$ of patients to 60 days following randomization (the anticipated follow-up period for the future definitive principal study). The authors were able to show that $85 \%$ of the prescribed continuous venovenous hemofiltration duration was delivered versus $88 \%$ in continuous venovenous hemodialysis; we have no information on the actual delivered dose. Also, interestingly, only $55 \%$ of the eligible patients (22\% of the screened population) were finally enrolled; one of the major merits of this study was to show that, in order to obtain significant clinical information from the difference between convection and diffusion, a larger trial with 700 patients per arm should probably be conducted, thus requiring a huge screening of about 6,000 patients. Clinical equipoise for such a trial, although long debated, is currently uncertain; the same group of researchers also performed a metaanalysis evaluating continuous hemofiltration versus hemodialysis in critically ill patients with severe AKI [31]: no effect on mortality and no impact on other clinical outcomes (RRT dependence in survivors, vasopressor use, organ dysfunction) was found from 16 randomized controlled studies comparing hemofiltration with hemodialysis. Interestingly, hemofiltration appeared to shorten the time to filter failure and increased clearance of medium to larger molecules, including inflammatory cytokines, compared with hemodialysis, although almost no studies measured changes in serum concentrations. Perhaps future studies should not necessarily research only the effects on mortality, but also the impact on other hard outcomes such as time to extubation, length of ICU stay, more specific details on hemodynamic impact, kidney recovery and other important follow-up variables (ICU readmission or other chronic organ failure).

As far as timing of RRT start is concerned, one should highlight that, currently, no standard definition of early or late dialytic therapy exists. As a matter of fact, we still have to clarify which RRT timing should be identified: does it depend on AKI pathogenesis? Should a decision be made by checking the time from ICU admission or the time from AKI diagnosis (according to standardized classifications or according to a specific stage)? Is a specific level of (positive) fluid balance an acceptable trigger 
for starting RRT? Otherwise, should we have in mind a specific target of negative fluid balance, and should RRT be started as soon as possible in order to reach such a goal? Does the severity of illness of AKI patients have a role in the timing decision? To tell the truth, each of these triggers has been proposed without evidence for significant improvement of patients' outcomes.

Based on the notion that the decision when to start RRT is a significant subjective component, Thakar and collaborators had the idea of conducting an international online survey on RRT timing among 172 nephrologists with a specific expertise in the field of critical care nephrology [32]. Interestingly, $53 \%$ of respondents indicated that there is no evidence in initiating early dialysis in AKI; in most cases this was due to the risk of early dialysis initiation outweighing the benefit. As a matter of fact, $46 \%$ of respondents instead indicated that they frequently initiate early dialysis. The survey also originally proposed three hypothetical case scenarios describing patients with different severity of critical illness: respondents clearly changed their recommendation for early dialysis as the predicted mortality of the cases increased: if two-thirds of all participants would start early dialysis in any case, almost the entirety of participants agreed with early RRT for the most severe patients. As far as clinical/biochemical triggers were concerned, blood urea nitrogen $<75 \mathrm{mg} / \mathrm{dl}$, relative increments of creatinine level (with respect to absolute values), and oxygen saturation (as an index of lung involvement) were preferred for cases at high risk of mortality. On the other hand, potassium levels, blood urea nitrogen levels $>75 \mathrm{mg} / \mathrm{dl}$ and absolute creatinine levels (in some answers, a serum creatinine $>5 \mathrm{mg} / \mathrm{dl}$ was indicated as a trigger) seemed more reasonable for low-risk patients. According to the authors' interpretation, RRT initiation in the ICU is still influenced by imminent indications rather than by a proactive decision based on the severity of AKI or azotemia. This interesting survey, different from another previous example [33,34], has the great merit of proposing different case scenarios in order to increase the complexity of clinical reasoning of respondents and to show, as a final result, realistic behavior of clinicians directly involved in clinical practice. Furthermore, the study showed that the absence of a clear consensus might be due to the lack of unequivocal evidence and perhaps due to the fact that this decision is complex and is associated with a significant level of subjectivity. As a final comment, one might add that, as all surveys, the prevalence of the respondents' original country, namely the United States, may significantly influence final results that cannot be generalized, for example, to European clinicians.

A call for an urgent need for trials focusing on RRT indications and timing is also the conclusion of the study by Clec'h and coworkers; manipulating data from the French longitudinal prospective multicenter Outcomerea database in a propensity analysis $(2,846$ critically ill patients), a comparison between critically ill patients treated with RRT and those not treated with RRT was conducted [35]. After heavy adjustments due to the comprehensible differences still present after matching, these authors showed that RRT was not associated with decreased mortality and even seemed to impair patients' outcome when initiated too late. An analog study aiming at the same primary aim, by Elseviers and colleagues, previously concluded that RRT was actually harmful for critically ill patients [36]. Detractors of this sort of observational statistical exercises may conclude that propensity analysis simply cannot be applied to match patients who needed RRT with others that did not need it. As a matter of fact, however, one must acknowledge that RRT absolute indications, and so dialysis timing, have never been standardized; no randomized trials have ever attempted the possibility of comparing RRT with socalled conservative management in selected patients. Apart from Clec'h and colleagues' and Elseviers and coworkers' provocative studies, results of such a trial might actually be surprising.

\section{Abbreviations}

AKI: Acute kidney injury; $\mathrm{Ca}^{\text {ion }}$ : Ionized calcium; CIN: Contrast-induced nephropathy; CRRT: Continuous renal replacement therapy; CT: Computed tomography; KDIGO: Kidney Disease: Improving Global Outcomes; RBF: Renal blood flow; RCA: Regional citrate anticoagulation; Rl: Resistive index; RIFLE: Risk, Injury, Failure, Loss of function, End-stage kidney disease RRT: Renal replacement therapy; T/I $\mathrm{Ca}^{2+}$ : Ionized calcium to total calcium.

\section{Competing interests}

The authors declare that they have no competing interests.

\section{Author details}

${ }^{1}$ Department of Pediatric Cardiosurgery, Bambino Gesù Children's Hospital, IRCCS, Piazza S. Onofrio 4, 00165, Rome, Italy. ${ }^{2}$ Department of Nephrology, Dialysis and Transplantation, S. Bortolo Hospital, Viale Rodolfi, 36100, Vicenza, Italy. ${ }^{3}$ International Renal Research Institute, Viale Rodolfi, 36100, Vicenza, Italy.

\section{Published: 22 Nov 2013}

\section{References}

1. Prowle J, Bagshaw SM, Bellomo R: Renal blood flow, fractional excretion of sodium and acute kidney injury: time for a new paradigm? Curr Opin Crit Care 2012, 18:585-592.

2. Lipcsey M, Bellomo R: Septic acute kidney injury: hemodynamic syndrome, inflammatory disorder, or both? Crit Care 2011, 15:1008.

3. Dewitte $A$, Coquin J, Meyssignac B, Joannès-Boyau O, Fleureau C, Roze $H$, Ripoche J, Janvier G, Combe C, Ouattara A: Doppler resistive index to reflect regulation of renal vascular tone during sepsis and acute kidney injury. Crit Care 2012, 16:R165.

4. Schnell D, Darmon M: Renal Doppler to assess renal perfusion in the critically ill: a reappraisal. Intensive Care Med 2012, 38:1751-1760.

5. Bragadottir G, Redfors B, Ricksten SE: Mannitol increases renal blood flow and maintains filtration fraction and oxygenation in postoperative acute kidney injury: a prospective interventional study. Crit Care 2012, 16:R159.

6. Ricci Z, Ronco C: New insights in acute kidney failure in the critically ill. Swiss Med Wkly 2012, 142:W13662. 
7. Hu JY, Meng XC, Han J, Xiang F, Fang YD, Wu J, Peng YZ, Wu YZ, Huang YS, Luo QZ: Relation between proteinuria and acute kidney injury in patients with severe burns. Crit Care 2012, 16:R172.

8. Mariano F, Camussi G: Unravelling the enigma of proteinuria in burn patients. Crit Care 2012, 16:184

9. Cely CM, Schein RM, Quartin AA: Risk of contrast induced nephropathy in the critically ill: a prospective, case matched study. Crit Care 2012, 16:R67.

10. KDIGO AKI Guideline Work Group, Lameire N, Kellum JA: Contrast-induced acute kidney injury and renal support for acute kidney injury: a KDIGO summary (Part 2). Crit Care 2013, 17:205.

11. Carmo LP DFd, Macedo E: Contrast-induced nephropathy: attributable incidence and potential harm. Crit Care 2012, 16:127.

12. Acute Dialysis Quality Initiative Workgroup, Bellomo R, Ronco C, Kellum JA, Mehta RL, Palevsky P: Acute renal failure - definition, outcome measures, animal models, fluid therapy and information technology needs: the Second International Consensus Conference of the Acute Dialysis Quality Initiative (ADQI) Group. Crit Care 2004, 8:R204-R212.

13. Acute Kidney Injury Network, Mehta RL, Kellum JA, Shah SV, Molitoris BA, Ronco C, Warnock DG, Levin A: Acute Kidney Injury Network: report of an initiative to improve outcomes in acute kidney injury. Crit Care 2007, 11: R31.

14. Wlodzimirow KA, Abu-Hanna A, Slabbekoorn M, Chamuleau RA, Schultz MJ Bouman CS: A comparison of RIFLE with and without urine output criteria for acute kidney injury in critically ill patients. Crit Care 2012, 16: R200

15. Ricci Z: RIFLE is alive: long live RIFLE. Crit Care 2012, 16:182.

16. KDIGO AKI Work Group: KDIGO clinical practice guideline for acute kidney injury. Kidney Int Suppl 2012, 2:1-138.

17. Kidney Disease: Improving Global Outcomes. www.kdigo.org.

18. KDIGO AKI Guideline Work Group, Kellum JA, Lameire N: Diagnosis, evaluation, and management of acute kidney injury: a KDIGO summary (Part 1). Crit Care 2013, 17:204.

19. Stein A, de Souza LV, Belettini CR, Menegazzo WR, Viégas JR, Costa Pereira EM, Eick R, Araújo L, Consolim-Colombo F, Irigoyen MC: Fluid overload and changes in serum creatinine after cardiac surgery: predictors of mortality and longer intensive care stay. A prospective cohort study. Crit Care 2012, 16:R99.

20. FINNAKI Study Group, Vaara ST, Korhonen AM, Kaukonen KM, Nisula S, Inkinen O, Hoppu S, Laurila JJ, Mildh L, Reinikainen M, Lund V, Parviainen I, Pettilä V: Fluid overload is associated with an increased risk for 90-day mortality in critically ill patients with renal replacement therapy: data from the prospective FINNAKI study. Crit Care 2012, 16:R197.

21. Program to Improve Care in Acute Renal Disease Study, Macedo $E_{1}$ Bouchard J, Soroko SH, Chertow GM, Himmelfarb J, Ikizler TA, Paganini EP, Mehta RL: Fluid accumulation, recognition and staging of acute kidney injury in critically-ill patients. Crit Care 2010, 14:R82.

22. Selewski DT, Cornell T, Blatt NB, Han YY, Mottes T, Kommareddi M, Gaies MG, Annich GM, Kershaw DB, Shanley TP, Heung M: Fluid overload and fluid removal in pediatric patients on extracorporeal membrane oxygenation requiring continuous renal replacement therapy. Crit Care Med 2012, 40:2694-2699.

23. Bellomo R, Cass A, Cole L, Finfer S, Gallagher M, Lee J, Lo S, McArthur C, McGuiness S, Norton R, Myburgh J, Scheinkestel C, Su S, RENAL Replacement Therapy Study Investigators: An observational study fluid balance and patient outcomes in the Randomized Evaluation of Normal vs. Augmented Level of Replacement Therapy trial. Crit Care Med 2012, 40:1753-1760

24. Chawla LS, Kellum JA, Ronco C: Permissive hypofiltration. Crit Care 2012, $16: 317$.

25. Ricci Z, Romagnoli S, Emma F: Permissive hypofiltration: an alternative view. Crit Care 2012, 16:458.

26. Morabito S, Pistolesi V, Tritapepe L, Zeppilli L, Polistena F, Strampelli E, Pierucci A: Regional citrate anticoagulation in cardiac surgery patients at high risk of bleeding: a continuous veno-venous hemofiltration protocol with a low concentration citrate solution. Crit Care 2012, 16:R111.

27. Schultheiß C, Saugel B, Phillip V, Thies P, Noe S, Mayr U, Haller B, Einwächter $\mathrm{H}$, Schmid RM, Huber W: Continuous venovenous hemodialysis with regional citrate anticoagulation in patients with liver failure: a prospective observational study. Crit Care 2012, 16:R162.

28. Link A, Klingele $M$, Speer T, Rbah R, Pöss J, Lerner-Gräber A, Fliser D, Böhm $M$ : Total-to-ionized calcium ratio predicts mortality in continuous renal replacement therapy with citrate anticoagulation in critically ill patients Crit Care 2012, 16:R97.

29. Oudemans-van Straaten HM, Kellum JA, Bellomo R: Clinical review: anticoagulation for continuous renal replacement therapy - heparin or citrate? Crit Care 2011, 15:202.

30. Wald R, Friedrich JO, Bagshaw SM, Burns KE, Garg AX, Hladunewich MA, House AA, Lapinsky S, Klein D, Pannu NI, Pope K, Richardson RM, Thorpe K, Adhikari NK: Optimal Mode of clearance in critically ill patients with Acute Kidney Injury (OMAKI) - a pilot randomized controlled trial of hemofiltration versus hemodialysis: a Canadian Critical Care Trials Group project. Crit Care 2012, 16:R205

31. Friedrich JO, Wald R, Bagshaw SM, Burns KE, Adhikari NK: Hemofiltration compared to hemodialysis for acute kidney injury: systematic review and meta-analysis. Crit Care 2012, 16:R146.

32. Thakar CV, Rousseau J, Leonard AC: Timing of dialysis initiation in AKI in ICU: international survey. Crit Care 2012, 16:R237.

33. Ricci Z, Ronco C, D'Amico G, De Felice R, Rossi S, Bolgan I, Bonello M, Zamperetti N, Petras D, Salvatori G, Dan M, Piccinni P: Practice patterns in the management of acute renal failure in the critically ill patient: an international survey. Nephrol Dial Transplant 2006, 21:690-696.

34. DOse REsponse Multicentre International collaborative Initiative (DO-RE-MI Study Group), Vesconi S, Cruz DN, Fumagalli R, Kindgen-Milles D, Monti G, Marinho A, Mariano F, Formica M, Marchesi M, René R, Livigni S, Ronco C: Delivered dose of renal replacement therapy and mortality in critically ill patients with acute kidney injury. Crit Care 2009, 13:R57.

35. Clec'h C, Darmon M, Lautrette A, Chemouni F, Azoulay E, Schwebel C, Dumenil AS, Garrouste-Orgeas M, Goldgran-Toledano D, Cohen Y, Timsit JF: Efficacy of renal replacement therapy in critically ill patients: a propensity analysis. Crit Care 2012, 16:R236.

36. SHARF investigators, Elseviers MM, Lins RL, Van der Niepen P, Hoste E, Malbrain ML, Damas P, Devriendt J: Renal replacement therapy is an independent risk factor for mortality in critically ill patients with acute kidney injury. Crit Care 2010, 14:R221

\section{$10.1186 /$ cc13126}

Cite this article as: Ricci and Ronco: Year in review 2012: Critical Care nephrology. Critical Care 2013, 17:246 